\title{
HEAD INJURIES AND MENINGITIS
}

BY

\author{
E. A. LINELL AND W. L. ROBINSON \\ From Divisions of Surgical Pathology and Neuropathology, University of Toronto \\ (ReCEIVED 11TH November, 1940)

\section{Introduction}

THE following cases have been selected from the files of the Division of Neuropathology in the University of Toronto to illustrate the types of head injury which may cause death from meningitis. The fatal infection of the meninges may occur at the time of the injury, killing the patient within a few days or weeks of the accident, but its onset may be delayed for years after the head injury. Three of the seven cases described below fall into the latter group, the patients having apparently recovered from their head injuries shortly after their accidents and having shown no symptoms until the sudden onset of a rapidly fatal meningitis.

The pneumococcus was the organism responsible for the meningitis in all these cases and, except for some variations mentioned in the text, the gross and microscopical pathological picture was typical of pneumococcal meningitis.

\section{Case Reports}

Case 1: M. W. (NP: 315-34), a man of 30 years, was probably kicked in the face by a horse on the 22nd October, 1934, although he could remember no details of the accident. He remained conscious but vomited several times during the next two days. He became unconscious on the 24th October and rapidly developed the signs of meningitis of which he died on the 26th October, four days after his accident.

Post mortem.-There was severe laceration of the face, with fractured nasal bones. Removal of the brain showed severe hæmorrhage covering the floor of the anterior cranial fossæ. The bone of these fossæ was shattered around the crista galli, which was loose, freely movable and displaced to the left. Scissors could be passed easily from the cranial cavity into the nasal fossæ. A fracture $3 \mathrm{~cm}$. long radiated outwards into the left orbital plate of the frontal bone.

There was greenish-yellow purulent exudate in the subarachnoid space. Pneumococci were cultured from this exudate and from the cerebrospinal fluid.

Comment.-This, then, is a case of rapidly fatal meningitis following an injury to the face of which the main external sign was simple fracture of the nasal bones.

Case 2: L. W. (NP: 170-36), a man of 50, was riding a bicycle when he was hit by a truck on the 21 st May, 1936 . On admission to hospital he was conscious but irritable. There was considerable bleeding from the back of the nose, with hæmatemesis. Leakage of cerebrospinal fluid from his nose was noted on 25th May. On the following day spinal puncture withdrew turbid fluid containing large numbers 
of polymorphonuclear leucocytes. His temperature rose till his death on the 27th May, six days after the accident.

Post mortem.-There was a large area of subcutaneous hæmorrhage on the left side of the scalp extending into the temporal muscle. Extradural hæmorrhages were found in the left anterior and middle cranial fossæ. A complicated fracture of the base of the skull began at the outer end of the left supra-orbital ridge. This extended backwards and inwards, crossing the midline through the body of the sphenoid bone and running along the antero-inferior border of the petrous portion of the right temporal bone to end in the right middle-ear cavity which was full of blood. A branch of this fracture extended inwards completely encircling the cribriform plate of the ethmoid and from here ran into the right frontal sinus which was pneumatizing the right orbital plate of the frontal bone. A second branch of the main fracture extended backwards and outwards across the left middle cranial fossæ to end in the base of the petrous portion of the left temporal bone. The sphenoidal and ethmoidal sinuses contained purulent exudate mixed with hæmorrhage.

Culture taken from the base of the brain yielded pneumococcus type 4 and there was well-marked purulent exudate in the subarachnoid space.

Comment.-This case is reported because it illustrates a basal skull fracture which had opened practically all the accessory nasal sinuses as well as both middle ear cavities. Purulent exudate in the frontal and ethmoidal sinuses was responsible for the meningitis in this case.

Case 3: R. T. (NP: 371-39), a boy of 3 years, was hit by a motor car shortly before his admission to hospital on the 17th September, 1939. Four days later he developed pneumococcal meningitis for which he received antipneumococcal serum combined with sulphapyridine. He gradually went down hill with persistence of his symptoms till his death on the 15 th October, about a month after the trauma. There was no clinical evidence of fracture of the skull.

Post mortem.-There was an area of extradural hæmorrhage in the right posterior cranial fossæ. This overlay a fracture which began slightly below and to the right of the external occipital protuberance. The fracture ran downwards and outwards to reach the petrous portion of the right temporal bone. It then ran inwards to end in the apex of this bone. Both middle-ear cavities showed evidence of chronic inflammation. There was also a mottled grey and dark-red thrombus in the lumen of the right lateral sinus in relation to the line of fracture. There was massive purulent exudate in the basal subarachnoid space.

Comment.-This case is of interest because the relationship of the headinjury to the meningitis was obscure until it was revealed at post mortem. It also illustrates the danger of chronic middle-ear disease in cases of fracture of the base of the skull. Finally the life of the patient was prolonged for nearly a month after the onset of meningitis, probably by the serum and sulphapyridine therapy.

Case 4: J. B. (NP: 164-37), a man of 49 years, was unconscious for 20 minutes following a fall downstairs on to a cement floor in the evening of the 12th February, 1937. On his recovery of consciousness he complained of severe frontal headache and he vomited. On admission to hospital the following day there was tenderness and œdema over the left parieto-occipital region of the scalp. His right knee-jerk was brisk and there was a doubtfully positive right Babinski. His cerebrospinal fluid pressure was high. There was no X-ray evidence of fracture of the skull.

Two days later, on the 15th February, he began to develop evidence of left otitis media, which required paracentesis of the drum on the 1st March. By the 11th March he showed a complete right hemiplegia with aphasia. On the following day an opera- 
tive exploration revealed an infected left subdural hæmatoma from the contents of which pneumococcus, Type 3 , was isolated. He improved temporarily but the development of a cerebral hernia obstructed efficient drainage of the infected subdural space and he gradually became more comatose, dying on the 24th April, $2 \frac{1}{2}$ months after his accident.

Post mortem.-A linear fracture, $10 \mathrm{~cm}$. long, ran horizontally through the lateral portion of the base of the occipital bone on the left side into the petrous portion of the left temporal bone. This bone was softened and contained purulent material.

The brain showed the infected left subdural hæmatoma, which had been opened and partially drained by operation. A horizontal section through this man's cerebral hemispheres is shown in Fig. 1.

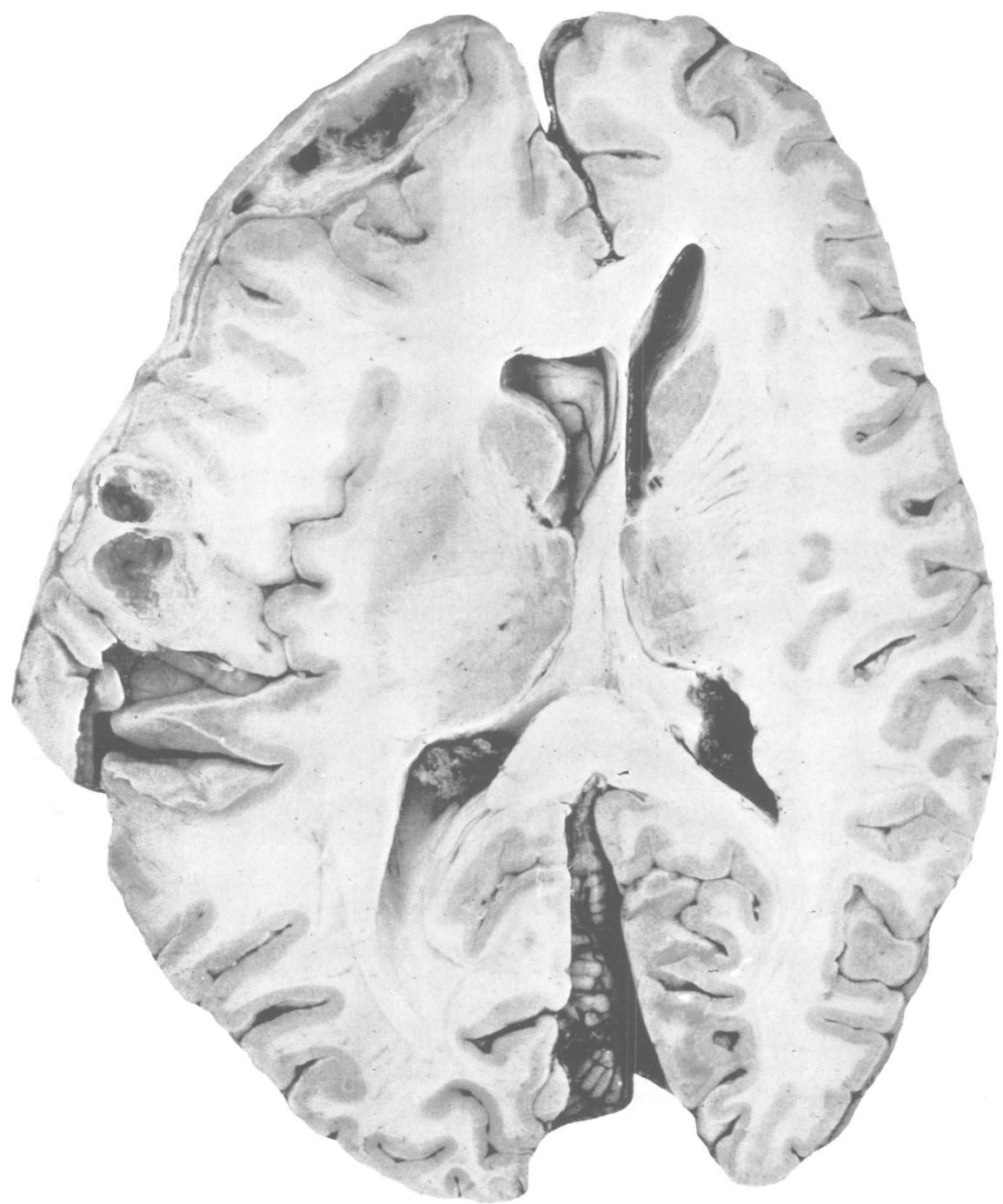

Fig. 1.-Photograph of a horizontal section of the cerebral hemispheres of Case 4, showing the area of herniation of the left cerebral hemisphere. The undrained portion of the old infected subdural hæmatoma is seen lying over the left frontal lobe. There are two small subcortical abscesses in the herniated tissue of the left fronto-parietal area. 
Comment.-This is a case in which infection from the patient's left middle-ear cavity and petrous temporal bone had been able to spread upwards through an unsuspected fracture line to infect a traumatic subdural hæmatoma, causing his death two and a half months after his fall.

Case 5: D. B. (NP: 176-39), was a professional jockey 22 years of age when he died on the 16th May, 1939. In 1934 he had had his nose broken by a horse. The skin laceration required sutures and he was in hospital for 11 days at that time. There were no significant after-effects.

On the 12th May, 1939, 5 years later, he had a slight cold in the head. Two days later he had a headache in the morning, by 3 p.m. he was vomiting and having convulsions. He was admitted to hospital comatose at 6 p.m. with all the signs of meningitis. The right maxillary and frontal sinuses were opaque to transillumination. Pneumococci were seen in a smear of his cerebrospinal fluid. In spite of vigorous treatment by soludagenan he died on the 16th May, four days after his first complaint of cold in the head.

Post mortem.-The left olfactory bulb and the adjacent frontal lobe were adherent to the cribriform plate of the ethmoid over the anterior ethmoidal air cells. The dura mater covering this area was hyperæmic and its inner surface had lost its lustre due to adhesion to the overlying leptomeninges and brain. The crista galli was deviated to

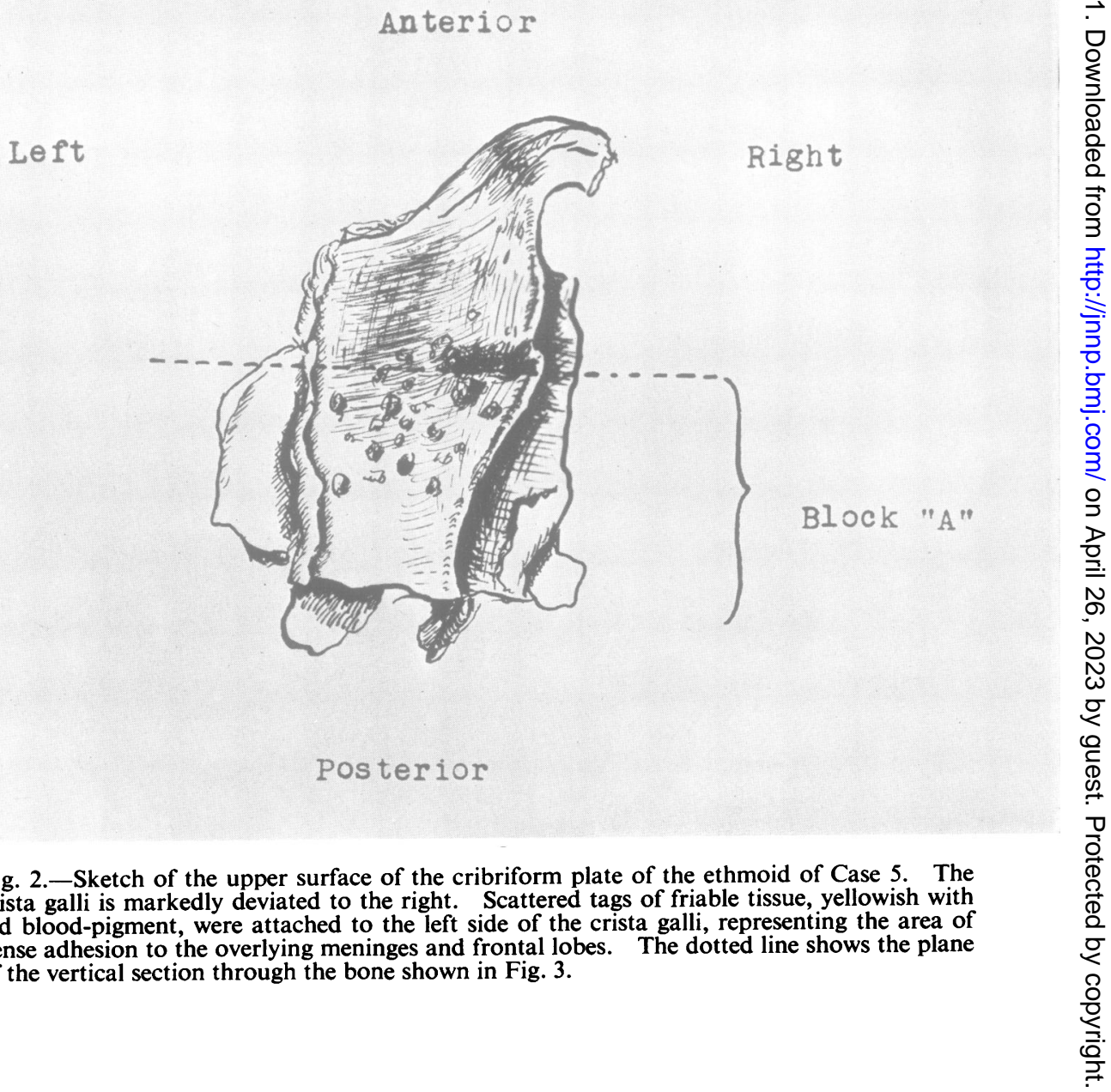


the right (Fig. 2), and there was old blood pigment covering the left side of the cribriform plate. There was pus in the right and left ethmoidal sinuses. Microscopical examination of a vertical section cut through the area of old trauma to the cribriform plate of the ethmoid in the plane of the dotted line shown in Fig. 2 is shown in Fig. 3. This demonstrates, under low magnification, the pathway of the patient's acute terminal pneumococcal infection from the roof of the right nasal fossæ to the overlying adherent leptomeninges.

There was no gross purulent exudate in the subarachnoid space, but widespread meningitis was seen microscopically.

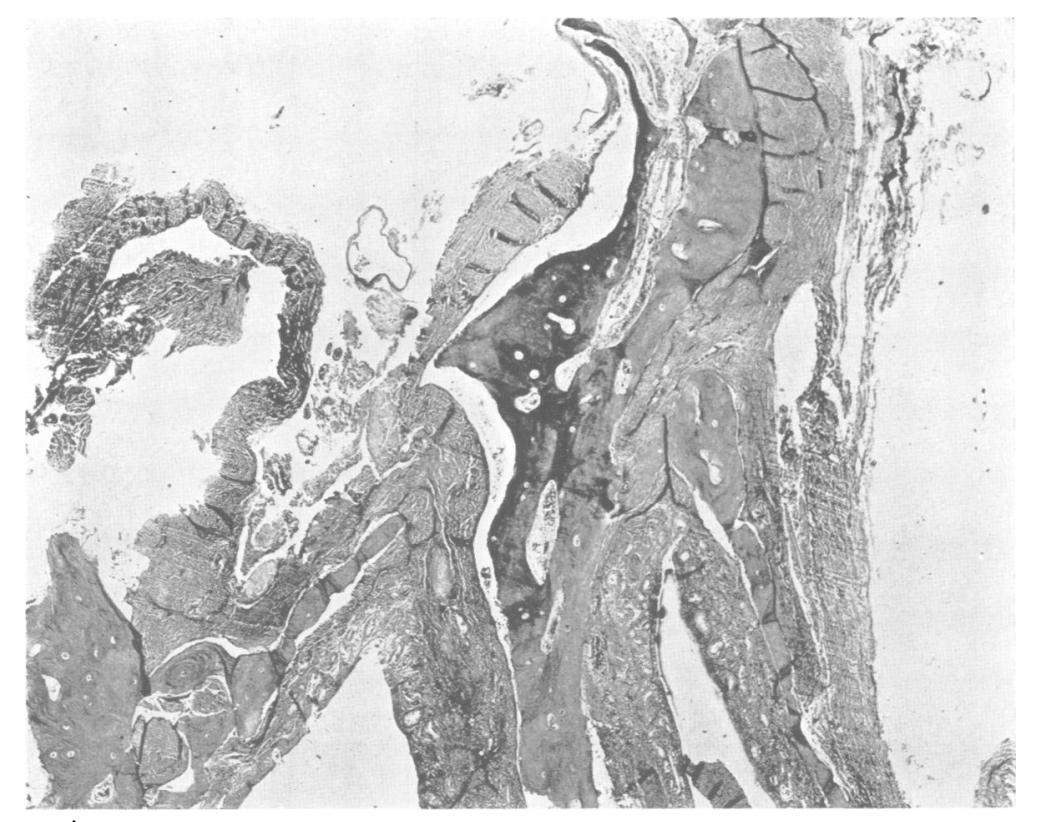

Fig. 3.-A low-power photomicrograph $(\times 14)$ of a vertical section through the cribriform plate of the ethmoid of Case 5. This shows the roof of the right nasal fossæ, to the left of the photograph, with the nasal septum between the two nasal cavities. It demonstrates the pathway of infection from the right nasal fossæ to the overlying, adherent leptomeninges.

Comment.-An illustration of an important type of case, further illustrated by Case 6 , in which an apparently trivial facial injury did sufficient damage to the cribriform plate of the ethmoid to provide a pathway for the passage of a pneumococcal nasal infection to the adjacent leptomeninges, causing death from a fulminating meningitis, five years after the original injury.

Case 6: W. P. (NP: 198-37), a man of 36 years at the time of his death, had an injury 14 years kefore, when he was hit in the midline of the forehead by the limb of a falling tree. He was unconscious for some hours and was off work for about two months. After this time he had complained of intermittent headaches often sufficiently severe to prevent his working.

In 1931 an X-ray showed a fracture near the midline of the vertical plate of the frontal bone extending obliquely downwards and outwards through the left frontal sinus and into the left ethmoidal cells. Late in May, 1937, he went to his doctor complaining of a foul-smelling nasal discharge. Increasing severity of headache necessitated his stopping work on the 4th June. He became confused and drowsy 
by the 6th June and was admitted to hospital in extremis on the following day. His cerebrospinal fluid on his admission was found to be opalescent and under greatly increased pressure. He died 17 hours later.

Post mortem.-The gross pathological findings were very similar to those recorded in Case 5. The orbital surface of the left frontal lobe was densely adherent in the region of the cribriform plate. An old fracture of the anterior cranial fossæ was found under this adherent area. It extended from the left optic foramen forwards and inwards to the cribriform plate and then turned backwards towards the right optic foramen. This fracture of the anterior cranial fossæ was considered to be a continuation of the frontal bone fracture seen by X-ray examination in 1931.

Seromucoid discharge was found in the sphenoidal and ethmoidal air sinuses. There was bronchopneumonia in the lower lobes of both lungs. Pneumococcus, Type 4, was cultured from the blood and from the cerebrospinal fluid. A vertical section cut to include the lines of old fracture of the cribriform plate of the ethmoid and of the right supra-orbital plate has been photographed under low magnification (Fig. 4). This shows an old fracture healed by fibrous tissue in the roof of the right

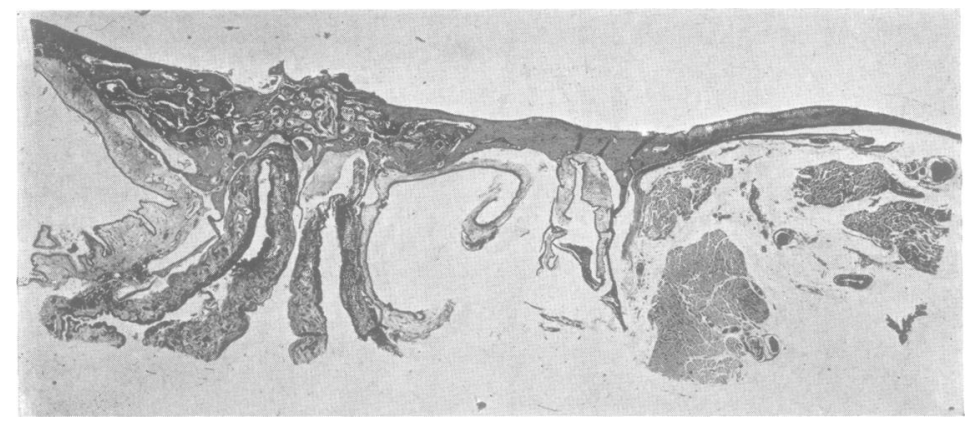

Fig. 4.-A low-power photomicrograph of the anterior cranial fossæ and adjacent structures of Case 6. This shows, from right to left, the right orbit, the right ethmoidal sinus, the nasal fossæ and the left ethmoidal sinus. In the plate of bone covering the right orbit an old fracture line can be seen extending through the thickness of the bone. Over the nasal fossæ another portion of the line of old fracture is shown by proliferated cancellous bone, with wide channels running through it.

orbit. This is shown under high magnification in Fig. 5. The second line of fracture, in the region of the cribriform plate, shows considerable proliferation of cancellous bone. This is shown under higher magnification in Fig. 6. Many of these cancellous spaces, especially those in relation with the infected left ethmoidal sinus, seen to the left of the photograph, contains large numbers of inflammatory cells (Fig. 7).

Comment.-In this case, therefore, it was concluded that the patient's terminal pneumococcal infection had spread from his acutely infected ethmoidal and sphenoidal sinuses through the site of a fourteen-year-old fracture in the cribriform plate of his ethmoid to the overlying, adherent leptomeninges, accounting for the meningitis of which he died. This viewpoint of the case was accepted by the Workmen's Compensation Board of Ontario and the widow was granted a pension for her husband's head injury.

Case 7: P. H. (NP: 31-34), a man of 52 years, died of a fulminating pneumococcal meningitis on the 15th January, 1934 . On the 27th September, 1931, he had shot himself accidentally with a $\cdot 22$ rifle. The bullet entered below the chin and passed upwards through the soft-palate just to the left of the midline. He did not lose consciousness. There were no positive neurological findings and no leakage of cerebro- 


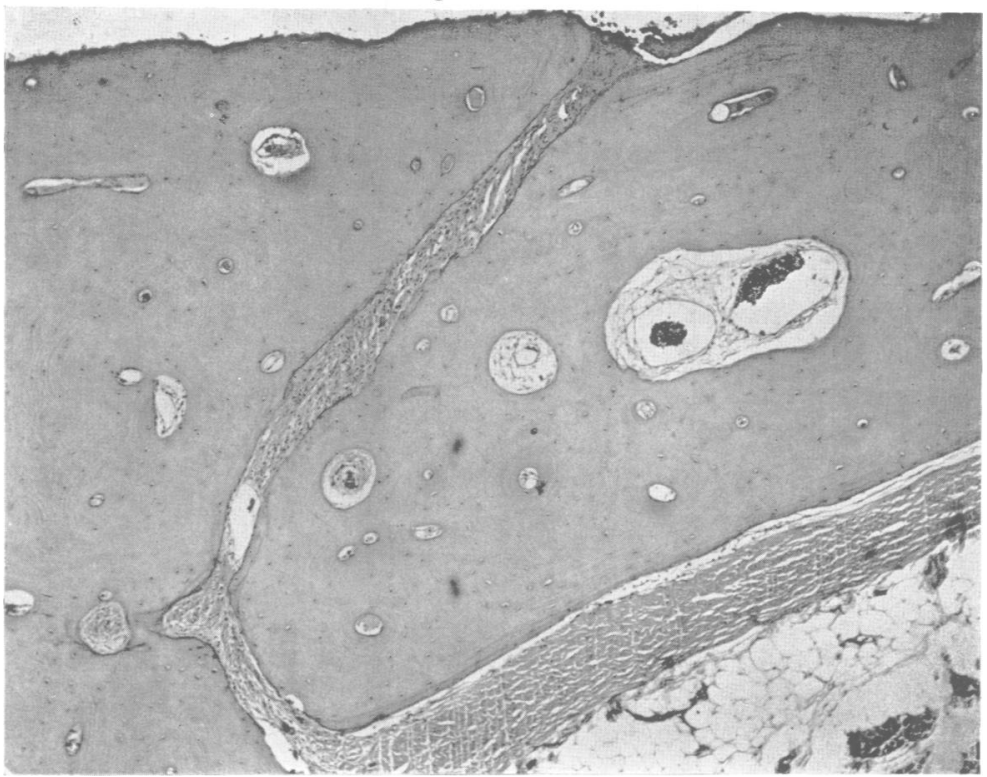

Fig. 5.-Higher magnification of the fracture line in the roof of the orbit in Case 6. This shows union of the fractured surfaces by fibrous tissue. The periosteum of the roof of the right orbit and orbital fat are shown in the lower part of the photograph.

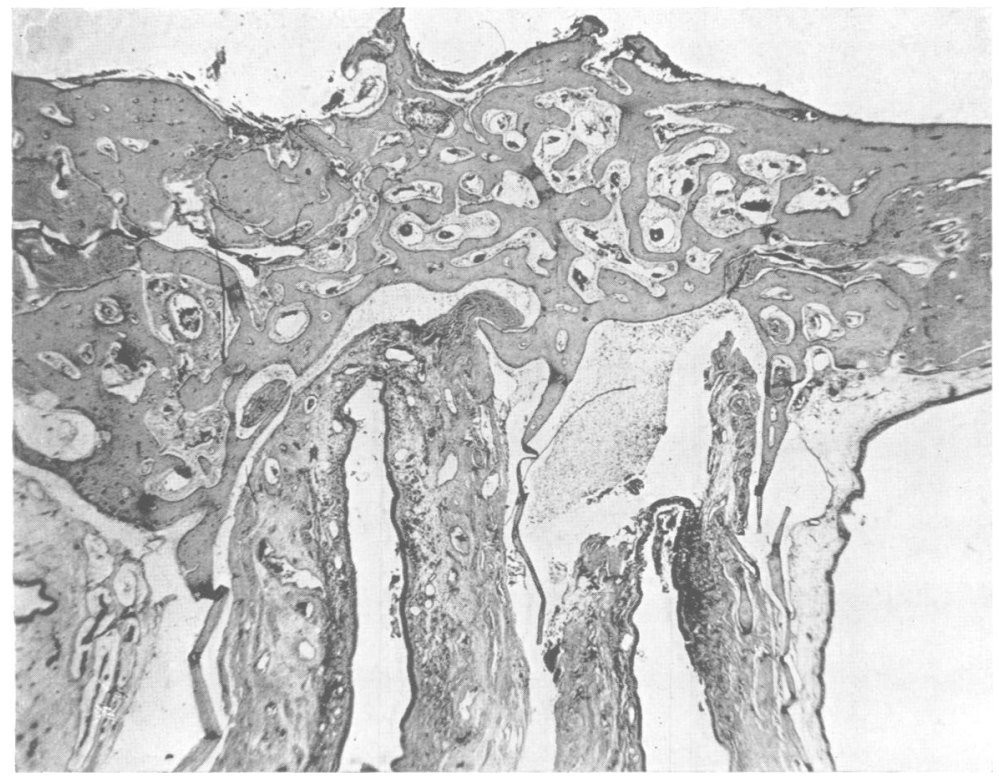

Fig. 6.-Proliferated cancellous bone at the site of the old fracture above the nasal fossæ of Case 6. Under this higher magnification inflamed and œdematous mucous membrane of the left ethmoidal sinus is seen to the extreme left of the photograph. The cancellous spaces in the bone overlying this sinus contain an acute inflammatory cellular reaction mixed with fibrous tissue (see Fig. 7). 


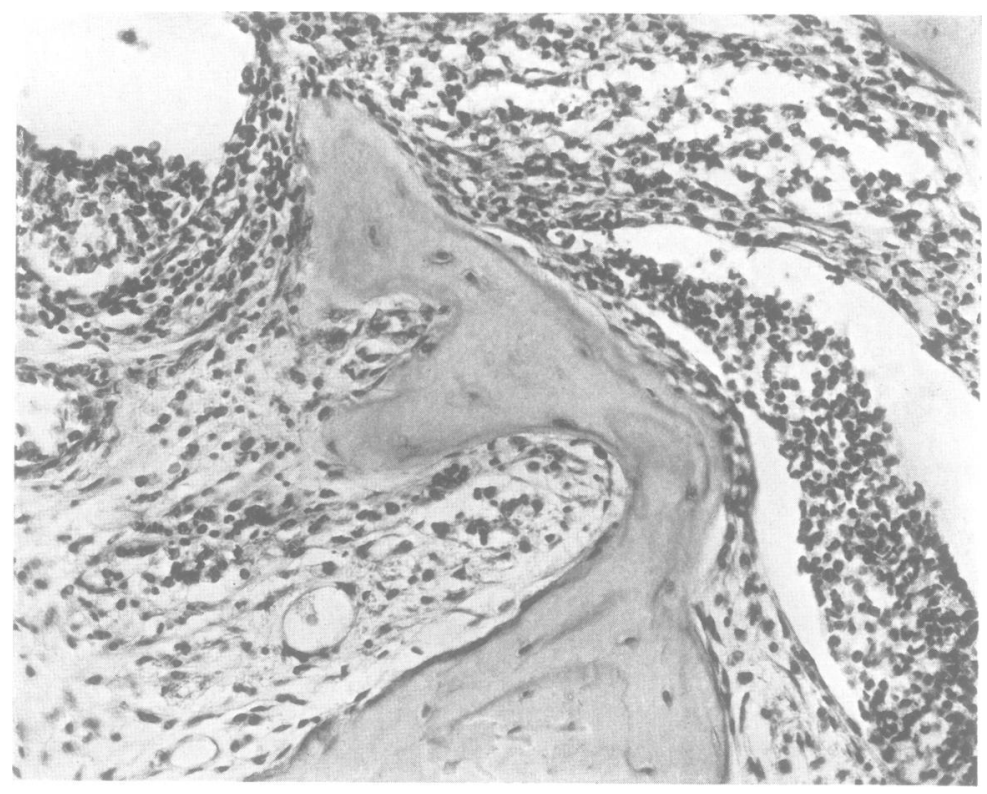

Fig. 7.-A higher power photomicrograph of the cancellous bone seen in Fig. 6, showing detail of the acute inflammatory reaction in the cancellous spaces at the site of a fourteen-yearold fracture. The terminal infection is believed to have reached the meninges from the acutely inflamed sinuses by this route.

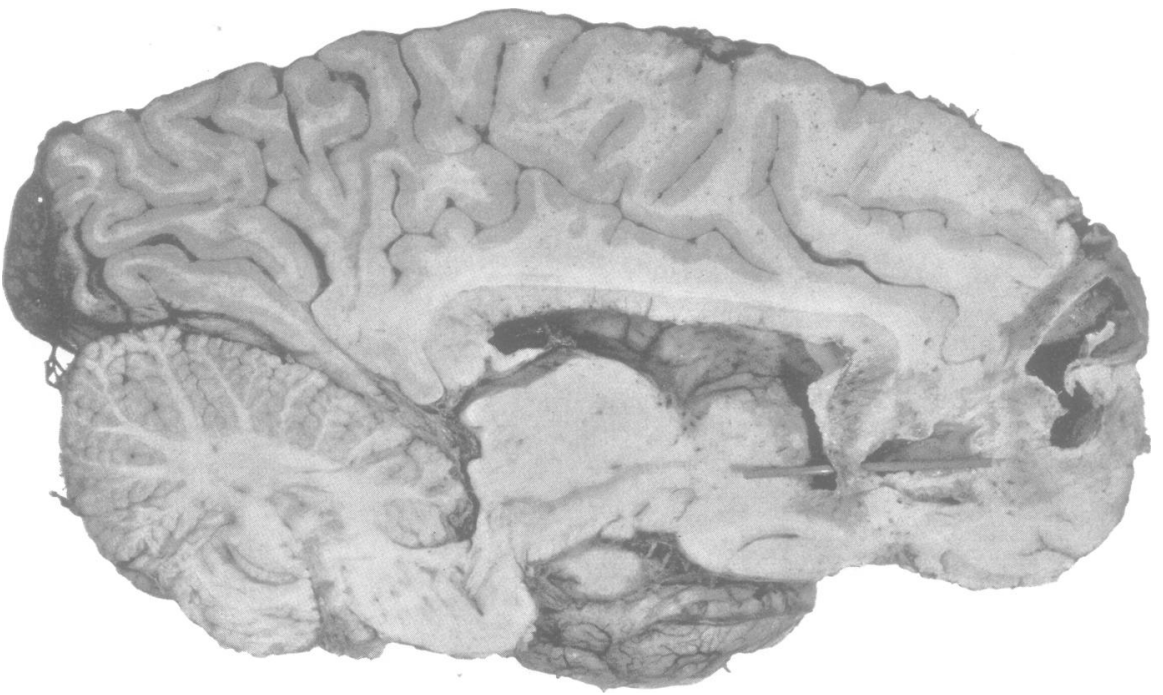

Fig. 8.-A para-sagittal section of the left cerebral hemisphere of Case 7, showing two pneumococcal abscesses in the line of the $2 \frac{1}{2}$-year-old bullet track. The lower abscess has ruptured into the anterior horn of the left lateral ventricle causing the patient's terminal ventricular and meningeal infection. 
spinal fluid. The bullet was seen by X-ray to be lying inside the vertex of the skull over the left frontal lobe just to the left of the superior sagittal sinus. On the following day the bullet was easily removed through a small left frontal bone-flap. The bullet track was explored with a soft rubber catheter which was passed from above through the left frontal lobe and through the bullet entry wound. After the operation the lower end of the catheter was seen by X-ray to be lying on the upper surface of the soft palate. It was gradually removed from below and the patient made an uninterrupted recovery.

He continued to work steadily and with no disability until a few days before his death, $2 \frac{1}{2}$ years later, when he developed a slight cold followed by a fulminating pneumococcal meningitis.

Post mortem.-The old bullet entry wound in the posterior part of the left orbital plate was covered by fibrous tissue. Its margin was continuous with a posterior ethmoidal air-cell. The bullet had passed upwards between the left optic nerve laterally and the left anterior cerebral artery mesially. The exit wound of the bullet from the brain was seen at the supero-mesial border of the left frontal lobe, $2.5 \mathrm{~cm}$. behind its pole. A para-sagittal section cut to the left of the midline in the line of the bullet track (Fig. 8), shows two abscesses in the left frontal lobe in the line of the bullet track. The lower abscess has broken into the cavity of the left lateral ventricle to produce the patient's terminal ventricular and meningeal infection.

Comment.-This case is of interest in suggesting that pneumococci had probably remained alive in the bullet-track for a period of two and a half years, during which time they gradually produced two asymptomatic brain abscesses in the patient's left frontal lobe. It is also intriguing to wonder whether the cause of the rupture of the abscess into the ventricle was the sudden increases of intracranial pressure, which probably occurred when he coughed and sneezed with his cold in the head. In this case there was no suggestion of a recent invasion of the cranial cavity by pneumococci from the nasal fossæ.

\section{Summary}

(1) Seven cases of meningitis are described in which the fatal infection followed a fracture of the base of the skull.

(2) In three of these cases the meningitis occurred 14, 5 and $2 \frac{1}{2}$ years, respectively, after injury. 